\title{
Assisting and advising complainants of sexual assault in the family planning setting
}

Deborah J Rogers, MMJ, DFF, Honorary Senior Lecturer in Forensic Medicine, St George's Hospital Medical School, London, UK

Correspondence: Dr D J Rogers, PO Box 66, Witney DO, OX29 4TD, UK. E-mail: djrogers@doctors.org.uk

(Accepted for publication $24^{\text {th }}$ April 2002)

\section{How to use a FACT}

A FACT is an up-to-date review of a subject relevant to the speciality, intended to help you fulfil your CPD requirements in your home or place of work. Whilst FACTs are edited and reviewed at various levels within the Faculty, the actual contents and views expressed are those of the authors and not the Faculty. More specifically, these reviews are not guidelines. The CEC is producing clinical guidelines separately.

FACTs have three sections: a review, a true/false test, and discussion points. To use a FACT to earn CPD credits you should do the following:

Working alone: Read the review and do the test on page 131. The answers are provided on page 139 so you can mark yourself. If there are points you are unsure about, disagree with, or need further clarification on, make a note of these for use at a later date. This should take you no more than 1 hour. Keep a record of having done this in your CPD diary and, unless indicated otherwise on the FACT, this will earn you 1 hour (DFFP), 1 credit (MFFP). Working as a group: Arrange a meeting of at least 1 hour with colleagues to discuss the discussion points given in the FACT (page 131) and any issues the participants have come up with as a result of reading the FACT. Keep a record of having done this in your CPD diary and, unless indicated otherwise on the FACT, this will earn you 1 hour (DFFP), 1 credit (MFFP).

\section{Introduction}

The latest Home Office crime statistics reveal that in the 12 months to March 2001 there were 5.2 million crimes recorded in England and Wales. During this period there were 37299 recorded sexual offences, $21 \%$ higher than in 1995-1996. ${ }^{1}$ Further analysis of the sexual offences data reveals that there were 7929 recorded rapes of a female within the 12 months ending March 2001; this represented a $1.5 \%$ rise compared to the previous year. It is likely that these figures represent only a small fraction of the sexual offences that actually take place. This review will examine how women who have been sexually assaulted may present to health care professionals working in primary care, family planning and genitourinary clinics, and discusses the management options that are available for such clients.

\begin{abstract}
Background
The Home Office have suggested that the increase in recorded sexual offences may be attributable to 'an increase in reporting by the public and changes in police practice'. 2 In the past decade the police have indeed worked hard at improving the services and facilities available to complainants of sexual assaults. Most areas in the UK have dedicated examination suites funded by the police. Typically these facilities have a reception room, a medical room and a bathroom for the complainant to use after the medical examination. The complainant is accompanied to the suite by a specially trained police officer and the examination is undertaken by a forensic medical examiner (police surgeon, forensic medical officer). Some urban areas (e.g. Manchester and Southeast London) are fortunate
\end{abstract}


enough to have a sexual assault referral centre that offers additional support and services to the complainant. In particular they accept referrals directly from the public and offer a forensic assessment, including anonymous sampling, for complainants who do not wish to contact the police at that stage. This is a distinct advantage since delays in reporting to the police normally result in the loss of any medical and forensic evidence.

International studies have shown that the majority of sexual assaults are not reported to the police. ${ }^{2}$ Some of these individuals may specifically seek help and advice for their immediate medical needs from health care services (e.g. family planning and genitourinary clinics); others may disclose the assault to a health care professional some time later when presenting to discuss other medical concerns. The following vignettes represent clients who presented to the author during one family planning session.

\section{Client 1}

A teenager attended the family planning clinic requesting emergency contraception. She said that she had been raped 2 days earlier by the brother of her best friend. During the consultation she revealed that her assailant was currently on early release from prison.

\section{Client 2}

A middle-aged mother of four attended the family planning clinic stating that she had missed a period. She said that 4 weeks earlier a family friend had raped her. Her husband had had a vasectomy. The pregnancy test was positive.

\section{Client 3}

A woman in her thirties attended the family planning clinic to discuss sexual problems including superficial dyspareunia. In the course of the consultation she disclosed that when she was a student she had been raped by a friend's boyfriend. She had developed genital warts as a consequence of the assault.

Every patient who presents following a sexual assault will have individual medical and forensic issues pertinent to their case. The following information will assist the practitioner in advising the patient.

\section{Relationship between the complainant and the assailant}

As demonstrated by the above cases the majority ( $88 \%$ ) of female rapes are committed by persons who are intimately known to the complainant (e.g. current or past sexual partner, family member, friend or work colleague) or who have a casual relation with the complainant (e.g. recently met at a party, provided a lift, client-prostitute). ${ }^{3}$ The remainder $(12 \%)$ are committed by total strangers. These have been categorised as 'intimate', 'acquaintance' and 'stranger' rape, respectively. ${ }^{4}$ The increase in reported rapes is accounted for by an increase in intimate and acquaintance rapes; the number of stranger rapes has not altered significantly in the past decade. ${ }^{3}$

Teenage complainants (age 13-15 years) are most likely to report having been raped by an acquaintance, often within the first 24 hours of meeting them. Although they usually met in a public outdoor place, the rapes were often alleged to have taken place in the assailant's home or some other private indoor place. ${ }^{3}$

\section{The nature of the sexual assault}

The term sexual assault is used to describe any nonconsensual sexual act. It encompasses both penetrative and non-penetrative sexual acts. Non-penetrative sexual acts include kissing the breasts, handling the genitalia, cunnilingus (mouth to female genitalia), anilingus (mouth the anus) and penile contact with the genitalia or anus that falls short of penetration. Penetrative sexual acts include penetration of the mouth, vagina and anus by a penis and penetration of the vagina and anus by an object. The former is known as fellatio. Rape is a legal term whose definition varies from jurisprudence to jurisprudence. English law defines rape as non-consensual peno-vaginal or peno-anal penetration. Fellatio and cunnilingus are classed as 'indecent assaults' under English law.

Peno-vaginal penetration is the most frequent component of the sexual assault cases that are reported by female complainants. ${ }^{5-7}$ Anal intercourse was reported by $6-26 \%$ of females who described having been sexually assaulted. ${ }^{5-7}$ Although anal intercourse may be the only sexual act that is performed, more frequently it is combined with vaginal and/or oral penetration. ${ }^{5,8}$ One study found that amongst 1507 (1403 females and 104 males) sexual assault cases referred to the Metropolitan Police Laboratory, $17 \%$ of female complainants and $14 \%$ of male complainants described performing fellatio during the sexual assault. Although fellatio may occur in isolation it occurs more frequently in conjunction with other sexual acts. ${ }^{8}$ As it may be difficult for complainants to describe peno-anal and peno-oral penetration it may be necessary to ask direct questions in a sensitive manner about these acts. ${ }^{8}$

\section{Immediate medical, psychological and social needs}

Clients who present to health care professionals following a recent sexual assault have a number of immediate medical, psychological and social needs, some of which they will have identified. These may include:

1. Emergency contraception

2. Prophylaxis against sexually transmitted infections (STIs) [including hepatitis B and human immunodeficiency virus (HIV)]

3. First aid for minor injuries

4. Investigation and treatment of more serious injuries

5. The support of a friend or family member

6. Replacement clothing (if still wearing the clothes worn at the time of the assault, as these will need to be retained)

7. Safe accommodation.

Once these immediate issues have been identified it is appropriate to enquire if the complainant has considered discussing the assault with the police. If the complainant wishes to report the sexual assault to the police, and forensic evidence might still be retrievable (see later), any medical intervention that could inadvertently remove any forensic evidence (e.g. fitting an emergency IUD) ideally should be deferred until after the forensic assessment has taken place.

Any information provided about the assault should be recorded verbatim. It is good practice to keep these details separate from the clinic notes in case a judge, or other presiding officer of a court, orders that the notes relating to the incident are disclosed to the court. If this practice is followed the clinic notes should state where the details of the assault have been recorded and stored.

\section{Disclosure to the police}

Competent adult and child complainants have a choice as to whether they report the assault to the police. Sexual assaults 
disclosed by complainants (adults or children) who lack the capacity to consent to disclosure need to be handled particularly sensitively. The General Medical Council (GMC) advises that in such cases information should be given promptly to 'an appropriate responsible adult or statutory agency, where you believe that the disclosure is in the patient's best interests. You should inform the patient that you intend to disclose the information before doing so. Such circumstances may arise in relation to children, where concerns about possible abuse need to be shared with other agencies such as social services. Where appropriate you should inform those with parental responsibility about the disclosure'?

Discussion of what the client will experience, including details of examinations required under the current police procedure, may help the client make an informed decision as to whether to disclose or not.

\section{Evidence-based forensic sampling}

The police need to be appraised of the nature and timing of the sexual assault in order to decide the extent and timing of any forensic medical examination. Various types of forensic evidence may be available such as:

- semen

- seminal fluid

- saliva

- fibres

- blood

- hairs

- condom lubricant.

Note that a distinction is made between semen and seminal fluid, as the assailant may have had a vasectomy.

It is appreciated that complainants may find the forensic assessment itself traumatic ${ }^{10,11}$ and, hence, they should not be subjected to speculative sampling that cannot be supported by the current forensic guidelines.

Spermatozoa may persist in the mouth for up to 31 hours. ${ }^{12}$ However, speed is of the essence for the collection of samples from the mouth, as most spermatozoa do not persist beyond 6 hours. ${ }^{12}$ Although drinking, cleaning the teeth, using a mouthwash, and so on, should be discouraged until after the samples have been obtained, these activities do not necessarily remove all spermatozoa. There are three recommended steps to sampling the oral cavity: ${ }^{13}$

1. The complainant is asked to produce $10 \mathrm{ml}$ of saliva into the appropriate container.

2. Two sequential swabs are rubbed over all surfaces in the mouth, including the cheeks, the dental margins, under the tongue and the palate.

3. The complainant is asked to use $10 \mathrm{ml}$ of sterile water as a mouthwash, which is retained as the third sample.

The order that these samples are obtained is important.

Provided there was vaginal ejaculation without a condom, spermatozoa should be found in the vagina for 24 hours after intercourse. However, they may be identified for up to 3 days and, occasionally, for up to 7 days. ${ }^{14,15}$ Therefore, samples are obtained from the female genitalia if the complainant presents within 7 days of peno-vaginal penetration, whether or not she believes that the male ejaculated. ${ }^{13}$ If a condom is used it is unlikely that spermatozoa will be present, although lubricant from a condom may be detectable for up to 30 hours. ${ }^{16}$ Whenever peno-vaginal penetration has occurred two vulval, two lowvaginal and two high-vaginal swabs should be obtained. Furthermore, as endocervical swabs have been shown to give a higher yield of spermatozoa if the sexual act was more than 48 hours earlier, then a single endocervical swab should also be obtained from complainants presenting between 2 and 7 days after the sexual assault. ${ }^{17}$ Only disposable specula should be used to obtain the high vaginal and endocervical swabs and these will be retained for forensic analysis. Sterile water may be used to lubricate the speculum; other lubricants should be avoided as they may decrease the amount of DNA that can be extracted from swabs (M Newton, Forensic Science Service, 2001, personal communication).

Spermatozoa have been identified on swabs taken from the anal canal/rectum 65 hours after peno-anal penetration. ${ }^{18}$ Defaecation does not necessarily remove the spermatozoa. ${ }^{12}$ Therefore, whenever peno-anal penetration has occurred two peri-anal, one anal canal and one rectal swab should be obtained. Typically a proctoscope is used to examine the anal canal and rectum for injuries and to facilitate obtaining internal swabs. As with the speculum the proctoscope should be disposable and retained for future forensic analysis, should this be necessary. Sterile water may be used to lubricate the proctoscope.

The skin should be sampled with wet or dry swabs (depending on the nature of the stain) if there is a possibility that it may be contaminated by body fluids from the assailant, e.g. saliva, semen, seminal fluid and blood. Skin samples should be obtained if the complainant has experienced cunnilingus or anilingus. Although no specific persistence data are available, washing does not necessarily remove all spermatozoa from the skin. ${ }^{19}$

Head hair and pubic hair are only cut or swabbed if they may be contaminated by body fluids from the assailant. However, any obvious foreign hairs or other material will be removed using single-use disposable forceps and the complainant's pubic hair is combed if there is a history of genital-to-genital contact. Research has shown that there is significant transfer of pubic hairs during vaginal intercourse. ${ }^{20}$ Although old forensic texts advocate obtaining plucked pubic hair as controls this is no longer considered necessary.

Similarly, fingernails are only sampled if the complainant describes scratching the assailant or if a fingernail has been broken during the incident and may remain at the scene or if the complainant does not recall what has happened and examination of the fingernails shows them to be macroscopically contaminated. The fingernails may be sampled either by simply scraping any debris from under the fingernails or by clipping the fingernails. The complainant's preference will be taken into account when deciding which method to use. There are no data available for how long forensic evidence persists under fingernails.

The police have special kits available that contain all the necessary swabs $(100 \%$ cotton with no transport medium in the sheaths), bottles, nail clippers, combs, forceps, scissors, sterile water and criminal justice-labelled bags for a forensic assessment. These kits have been designed to ensure the best quality evidence is collected and to minimise challenges regarding cross-contamination. All samples have to be labelled, signed, sealed and stored in a specific way to ensure that they comply with legal requirements. A number of control samples also have to be collected. Swabs and bottles available from the health service are not suitable for obtaining or storing forensic samples.

If the sexual assault results in pregnancy, and the complainant has a therapeutic abortion, with her consent the products of conception may be retained and analysed to determine the paternity of the fetus. Following the abortion the products of conception should be placed into a plain container that has not been used for anything else. For 
aesthetic reasons the container may then be placed into an opaque bag prior to handing it to a police officer. The police officer will label it and either transport it immediately to a forensic science laboratory or arrange for it to be frozen. Control samples will need to be obtained from the woman and from the purported father to determine their DNA profiles.

\section{Medical examination}

In all cases the complainant will be offered a complete general medical examination to document any injuries, stains or marks and to note any diseases or signs of old trauma that may affect the interpretation of the medical findings. The extent of the ano-genital examination will be determined by the nature of the allegation, e.g. if the complainant only describes performing fellatio there is no indication to examine the ano-genital area. Throughout the examination the dignity of the complainant will be respected and they will be reminded that they have the right to decline any part of the proposed examination. All findings will be detailed precisely in contemporaneous medical records and on occasions photodocumentation may be used.

\section{Substance-facilitated sexual assault}

Substance- or drug-facilitated sexual assault is the term used to define offences in which complainants are 'subjected to non-consensual sexual acts while they are incapacitated or unconscious due to the effects of alcohol and/or drugs, and therefore prevented from resisting and/or are unable to consent'. ${ }^{21}$ The substance may have been consumed voluntarily by the complainant or administered surreptitiously by the assailant. Alcohol is the substance most commonly detected by toxicological analysis of urine taken from complainants of substance-facilitated sexual assault. ${ }^{22}$ It is rare to find evidence of any other drugs with a sedative action, although this could be because of delays in obtaining the relevant samples. ${ }^{23}$ Ideally a sample of urine should be obtained from a complainant within a few hours of the alleged substance-facilitated sexual assault. However, current guidelines state that a sample of blood $(10 \mathrm{ml}$ placed in a bottle with a preservative and an anti-coagulant) and a sample of urine ( $20 \mathrm{ml}$ placed in a bottle with a preservative) should be requested from the complainant if the offence is reported within 4 days; urine alone is requested if the offence is reported between 4 and 7 days. ${ }^{13}$ Recent research has found that analysis of hair may be useful in detecting single doses of some benzodiazepines. ${ }^{24}$

Although any sedative drugs can be used to facilitate a sexual assault, the ones that have received most press attention are Rohypnol (flunitrazepam) and gammahydroxybutyrate. In response to public concerns regarding its use in substance-mediated sexual assaults Rohypnol is no longer available on an NHS prescription and the tablets have been reformulated so that when dissolved in a liquid they colour it blue. By comparison, gammahydroxybutyrate is available from gyms, dance clubs, sex shops and recipes on the Internet. ${ }^{25}$ Liquid gammahydroxybutyrate is colourless, odourless and, in its pure form, almost tasteless (non-pure forms have a salty or soapy taste). ${ }^{25}$ Gamma-hydroxybutyrate can induce rapid unconsciousness, particularly if combined with alcohol, and, like benzodiazepines, it is associated with amnesia. Hence, some experts now consider gammahydroxybutyrate as being the drug most favoured by rapists. $^{25}$

\section{Aftercare}

Whether or not the complainant discloses the sexual assault to the police he/she will need advice regarding follow-up in relation to emergency contraception, STI testing, and the local facilities for counselling. If the sexual assault has exposed the complainant to a high risk of acquiring hepatitis B or HIV (e.g. if the assailant had obvious risk factors for hepatitis B or HIV or if they had been assaulted by multiple assailants) the doctor should seek urgent advice from a physician experienced in the management of hepatitis B and HIV exposure. For optimal efficiency hepatitis B prophylaxis should be commence within 72 hours of the at-risk exposure. Ideally postexposure prophylaxis for HIV should be commenced within a few hours of the assault but it may be effective if started after longer time intervals. Advice on these matters can be obtained from an expert in genitourinary medicine in working hours, and either the local accident and emergency (A\&E) department or the doctor on-call for public health out of hours.

In all other cases the complainant should be advised to attend a genitourinary clinic 14 days after the assault where he/she will be offered an STI screen. This timescale takes account of the incubation period of the most common STIs (Table 1); however, an earlier visit may be helpful for advice and counselling. A special appointment can be made by liaison with a health advisor at the complainant's local genitourinary clinic.

With the complainant's consent his/her general practitioner (GP) should be appraised of the allegation.

Table 1 Incubation period of common sexually transmitted infections ${ }^{26}$

Sexually transmitted infection (STI) Incubation period (days)

\begin{tabular}{lc}
\hline Neisseria gonorrhoeae & $2-7$ \\
Herpes simplex virus & $2-12$ \\
Trichomonas vaginalis & $4-20$ \\
Chlamydia trachomatis & $7-14$ \\
Treponema pallidum & $14-84$ \\
Human immunodeficiency virus (HIV) & $30-90$ \\
Human papilloma virus & $30-140$ \\
Hepatitis B virus & $45-180$ \\
\hline
\end{tabular}

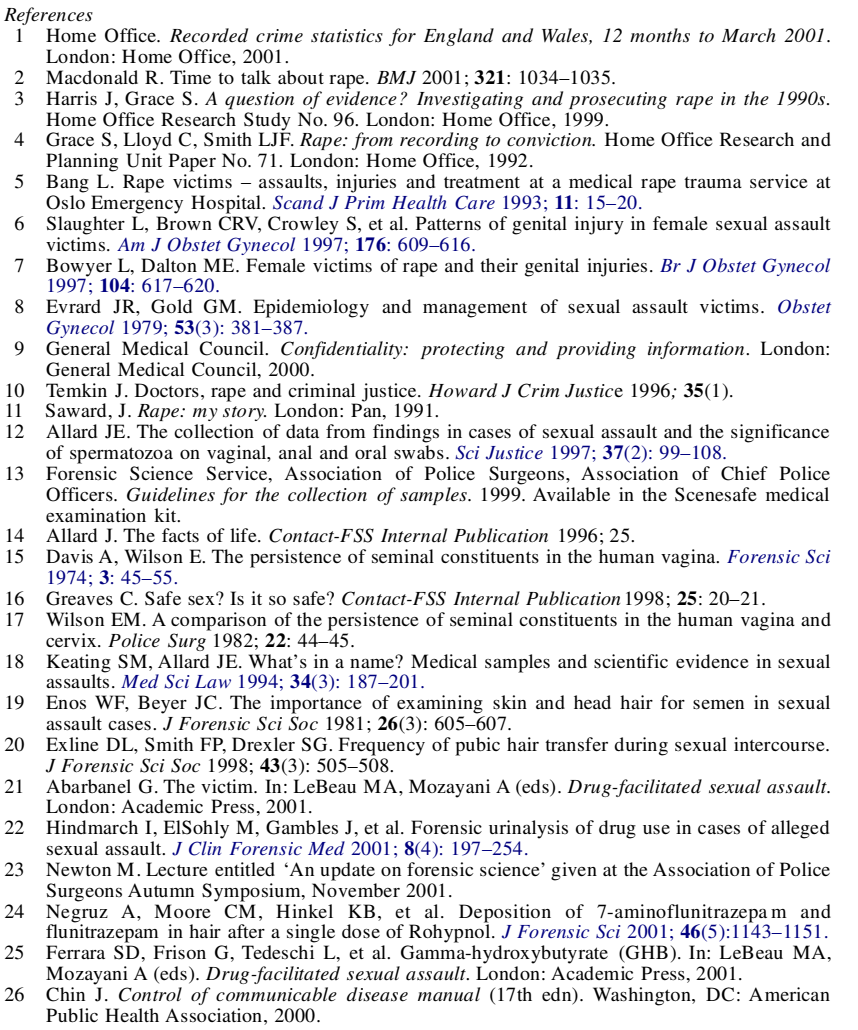


Discussion points

1. Devise a protocol for your family planning clinic for dealing with complainants of sexual assault who are under 16 years of age and those who are over 16 years of age.

2. What facilities exist locally for complainants who report to the police?

3. Who should fund sexual assault referral centres?

4. What are the local policies for sexually transmitted disease prophylaxis in relation to complainants of sexual assault?

5. List the local counselling services available for complainants of sexual assault.

\begin{tabular}{ll}
\hline F & Faculty \\
A & Aid to \\
C & CPD \\
T & Topics
\end{tabular}

Review No. 2002/03

A CPD Self-Assessment Test QUESTION SHEET

\section{Assisting and advising complainants of sexual assault in the family planning setting}

Indicate your answer by ticking the appropriate box for each question

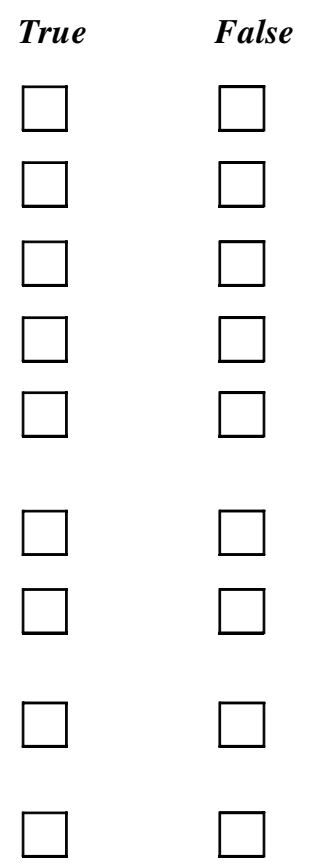

9. Alcohol is the most common substance detected on toxicological analysis of urine samples from complainants of sexual assault.

10. There is no point obtaining samples for toxicology if a complainant presents more than 48 hours after a possible substance-facilitated sexual assault.

\section{Visit the Faculty Website at: www.ffprhc.org.uk}

